\title{
Pairwise Intersections and Forbidden Configurations
}

\author{
R. P. Anstee ${ }^{1 \dagger}$ and Peter Keevash ${ }^{2 \ddagger}$ \\ ${ }^{1}$ Mathematics Department, The University of British Columbia, Vancouver, B.C., Canada V6T $1 Z 2$ \\ ${ }^{2}$ Department of Mathematics, California Institute of Technology Pasadena, California, USA
}

Let $f_{m}(a, b, c, d)$ denote the maximum size of a family $\mathcal{F}$ of subsets of an $m$-element set for which there is no pair of subsets $A, B \in \mathcal{F}$ with

$$
|A \cap B| \geq a, \quad|\bar{A} \cap B| \geq b, \quad|A \cap \bar{B}| \geq c, \quad \text { and } \quad|\bar{A} \cap \bar{B}| \geq d .
$$

By symmetry we can assume $a \geq d$ and $b \geq c$. We show that $f_{m}(a, b, c, d)$ is $\Theta\left(m^{a+b-1}\right)$ if either $b>c$ or $a, b \geq 1$. We also show that $f_{m}(0, b, b, 0)$ is $\Theta\left(m^{b}\right)$ and $f_{m}(a, 0,0, d)$ is $\Theta\left(m^{a}\right)$. This can be viewed as a result concerning forbidden configurations and is further evidence for a conjecture of Anstee and Sali. Our key tool is a strong stability version of the Complete Intersection Theorem of Ahlswede and Khachatrian, which is of independent interest.

Keywords: forbidden configurations, extremal set theory, intersecting set systems, uniform set systems, $(0,1)$ matrices

Let $f_{m}(a, b, c, d)$ denote the maximum size of a family $\mathcal{F}$ of subsets of an $m$-element set for which there is no pair of subsets $A, B \in \mathcal{F}$ with

$$
|A \cap B| \geq a, \quad|\bar{A} \cap B| \geq b, \quad|A \cap \bar{B}| \geq c, \quad \text { and } \quad|\bar{A} \cap \bar{B}| \geq d .
$$

By symmetry we can assume $a \geq d$ and $b \geq c$.

Theorem 1 Suppose $a \geq d$ and $b \geq c$. Then $f_{m}(a, b, c, d)$ is $\Theta\left(m^{a+b-1}\right)$ if either $b>c$ or $a, b \geq 1$. Also $f_{m}(a, 0,0, d)$ is $\Theta\left(m^{a}\right)$ and $f_{m}(0, b, b, 0)$ is $\Theta\left(m^{b}\right)$.

Some motivation for studying this function comes from the forbidden configuration problem for matrices popularised by the first author. We can identify a family $\mathcal{A}=\left\{A_{1}, \cdots, A_{n}\right\}$ of subsets of $[m]$ with an $m \times n(0,1)$-matrix $A$ determined by incidence, i.e. $A_{i j}$ is 1 if $i \in A_{j}$, otherwise 0 . Such a matrix is simple, by which we mean it has no repeated columns. Let $F$ be a $(0,1)$-matrix (not necessarily simple). We define forb $(m, F)$ to be the largest $n$ for which there is a simple $m \times n(0,1)$-matrix $A$ that does not contain an $F$ configuration, i.e. a submatrix which is a row and column permutation of $F$. If we interpret

\footnotetext{
${ }^{\dagger}$ Research is supported in part by NSERC

$\ddagger$ Research was done partly while visiting the first author with the support of the first author’s NSERC grant 
$A, F$ as incidence matrices of systems $\mathcal{A}, \mathcal{F}$ (the latter possibly having sets with multiplicity) then $A$ has an $F$ configuration exactly when $\mathcal{A}$ has $\mathcal{F}$ as a trace, i.e. $\mathcal{F} \subset\{A \cap X: A \in \mathcal{A}\}$ for some $X \subset[m]$.

The first forbidden configuration result was obtained independently by Sauer [6], Perles, Shelah [7], Vapnik and Chervonenkis [8]. When $F$ is the $k \times 2^{k}(0,1)$-matrix with all possible distinct columns they showed that forb $(m, F)=\sum_{i=0}^{k-1}\left(\begin{array}{c}m \\ i\end{array}\right)$. For a general $k$-row matrix $F$, Füredi obtained an $O\left(m^{k}\right)$ upper bound on forb $(m, F)$, but it seems hard to determine the order of magnitude of forb $(m, F)$ for each $F$. This was achieved when $F$ has 2 rows by Anstee, Griggs and Sali [2] and for 3 rows by Anstee and Sali [3], but is open in general.

It is not hard to see that if $F$ consists of a single column with $s$ 's and $t 1$ 's then forb $(m, F)$ is $\Theta\left(m^{\max \{s-1, t-1\}}\right)$. In this paper we solve the problem when $F$ has two columns. Let $F_{a b c d}$ be the $(a+b+c+d) \times 2(0,1)$-matrix which has $a$ rows of [11], $b$ rows of [10], $c$ rows of [01], $d$ rows of [00]. Then forb $\left(m, F_{a b c d}\right)=f_{m}(a, b, c, d)$ as defined above.

In [3] a conjecture was made for the asymptotic behaviour of forb $(m, F)$ as a function of $m$ and $F$. In particular, a restricted set of constructions of simple matrices were described in [3] that were conjectured to predict the asymptotics of forb $(m, F)$. These were used in this paper to predict the asymptotics in Theorem 1 as well as to provide construction. This is further evidence for the conjecture in [3].

Our key tool is a strong stability version of the Complete Intersection Theorem of Ahlswede and Khachatrian [1], which is of independent interest. Strong stability results have been employed with success by the second author, for example in [4],[5]. First we recall some notation. Let numbers $k, r_{1}, r_{2}$ be given and suppose $G$ and $H$ are disjoint sets with $|G|=k-r_{1}+r_{2}$. We define $\mathcal{I}_{r_{1}, r_{2}}^{k}$ on the pair $(H, G)$ to be the family consisting of all sets of size $k$ in $G \cup H$ that intersect $G$ in at least $k-r_{1}=|G|-r_{2}$ points. Note that any two sets in $\mathcal{I}_{r_{1}, r_{2}}^{k}$ have at least $|G|-2 r_{2}=k-r_{1}-r_{2}$ points in common, i.e. $\mathcal{I}_{r_{1}, r_{2}}^{k}$ is $(k-r)$-intersecting, where $r=r_{1}+r_{2}$.

We also define $\mathcal{F}_{r_{1}, r_{2}}^{k}$ on the pair $(H, G)$ to be the family consisting of all sets of size $k$ in $G \cup H$ that intersect $G$ in exactly $k-r_{1}=|G|-r_{2}$ points. Clearly this is a subsystem of $\mathcal{I}_{r_{1}, r_{2}}^{k}$ and $\left|\mathcal{I}_{r_{1}, r_{2}}^{k} \backslash \mathcal{F}_{r_{1}, r_{2}}^{k}\right|$ is of a lower order of magnitude than $\left|\mathcal{I}_{r_{1}, r_{2}}^{k}\right|$ and $\left|\mathcal{F}_{r_{1}, r_{2}}^{k}\right|$. In particular, if the systems are defined on the ground set $[m]$ with $k=\Theta(m)$ then $\left|\mathcal{I}_{r_{1}, r_{2}}^{k}\right|$ and $\left|\mathcal{F}_{r_{1}, r_{2}}^{k}\right|$ are $\Theta\left(m^{r}\right)$, whereas $\left|\mathcal{I}_{r_{1}, r_{2}}^{k} \backslash \mathcal{F}_{r_{1}, r_{2}}^{k}\right|<m^{r-2}$. The Complete Intersection Theorem, conjectured by Frankl, and proved by Ahlswede and Khachatrian [1], is that any $k$-uniform, $(k-r)$-intersecting family of maximum size on a given ground set is isomorphic to $\mathcal{I}_{r-p, p}^{k}$, for some $0 \leq p \leq r$, which depends on the size of the ground set. We prove the following result.

Theorem 2 Suppose $\mathcal{A}$ is a $k$-uniform $(k-r)$-intersecting set system on $[m]$ of size at least $(5 r)^{5 r} m^{r-1}$. Then $\mathcal{A} \subset \mathcal{I}_{r-p, p}^{k}$ for some $0 \leq p \leq r$.

We use this theorem in our proofs of the upper bounds in Theorem 1 in cases where $\mathcal{A}$ is a $k$-uniform $(k-r)$-intersecting set system satisfying some additional properties. If $|\mathcal{A}|$ is small, we can ignore it for the purposes of upper bounds. If $|\mathcal{A}|$ is large enough to matter for the upper bounds, we can use the fact that $\mathcal{A} \subset \mathcal{I}_{r-p, p}^{k}$ to deduce structure in $\mathcal{A}$ (e.g. the partition $G, H$ above) which we can exploit in our proofs.

\section{References}

[1] R. Ahlswede and L.H. Khachatrian, The complete intersection theorem for systems of finite sets, European J. Combin. 18 (1997), 125-136. 
[2] R.P. Anstee, J.R. Griggs and A. Sali, Small Forbidden Configurations, Graphs and Combinatorics, 13 (1997), 97-118.

[3] R.P. Anstee and A. Sali, Small Forbidden Configurations IV: The 3-rowed case, Combinatorica, to appear.

[4] P. Keevash and D. Mubayi, Stability theorems for cancellative hypergraphs, J. Combin. Theory Ser. B 92 (2004), 163-175.

[5] P. Keevash and B. Sudakov, The Turán number of the Fano plane, Combinatorica, to appear.

[6] N. Sauer, On the density of families of sets, J. Combin. Th. Ser A 13(1973), 145-147.

[7] S. Shelah, A combinatorial problem: Stability and order for models and theories in infinitary languages, Pac. J. Math. 4(1972), 247-261.

[8] V.N. Vapnik, A.Ya. Chervonenkis, On the uniform convergence of relative frequencies of events to their probabilities, Th. Prob. and Applics. 16(1971), 264-280. 
